\title{
A SPONTANEOUS EPIDEMIC AMONG LABORATORY RABBITS CAUSED BY A PARATYPHOID \\ B. BACILLUS RELATED TO THE RODENT GROUP
}

\author{
V. M. LITCH AND K. F. MEYER \\ From the George William Hooper Foundation for Medical Research, University of \\ California, San Francisco, Calif.
}

Spontaneous bacterial infections among laboratory or breeding rabbits are not uncommon. Irrespective of the confusing nomenclature, ${ }^{1}$ which may at first suggest a variety of microbian causes, the etiology of such epidemics is in our experience usually confined to $\mathrm{B}$. cuniculisepticus and B. bronchisepticus. Recently our attention was called to the occurrence of true paratyphoid $B$. infections.

According to the available information, epidemics among rabbits caused by this group of organisms are rare. Uhlenhuth and Hübner ${ }^{2}$ mention, in their summary on the paratyphoid group, that Holst and Hottinger determined bacteria of this group to be the cause of spontaneous epizootics among rabbits. Also Pfeiler ${ }^{3}$ expresses the same idea in a short sentence in one of his recent reviews. A perusal of the articles of Holst and Hottinger referred to did not inform us of the facts on which these statements are based. In his complete review Loele ${ }^{4}$ does not mention Holst and Hottinger. A definite description of paratyphoid fever in rabbits as a disease entity is therefore not available although Morgan ${ }^{5}$ obtained three cultures of an organism like paratyphoid A from rabbits' feces, an observation that we were able to confirm. In 1914 Ferry ${ }^{B}$ made an intensive study of the causative organisms found in B. bronchisepticus epidemics among laboratory animals. In a few cases he was able to isolate an organism of the partyphoid B. enteritidis group; he considered these bacteria to be secondary invaders superimposed on an infection with $B$. bronchisepticus or B. cuniculisepticus. Krumwiede, Valentine and Kohn ${ }^{\top}$ have recently published an article in which they show that paratyphoid strains isolated from guineapigs, mice, rabbits and cats differ antigenically based on specific absorption tests from human paratyphoid B. or B. schottmülleri-strains. A distinct type or group, the so-called "rodent paratyphoid," is according to their views encountered in spontaneous infections of laboratory animals, especially rodents.

Received for publication September 2, 1920.

1 Hutyra and Mareck: Spezielle Pathologie \& Therapie d. Haustiere, 1913, 1, p. 104; Ferry and Hoskins: Jour. Lab. \& Clin. Med., 1920, 5, p. 311.

Kolle and Wassermann: Handb. d. pathog. Mikroorganis., 1913, 3, p. 1102.

8 Friedberger and Pfeiffer: Lehrbuch d. Mikrobiologie, 1919, 2, p. 917.

- Ergebn. d. allg. Pathol. u. Pathol. Anatomie, 1915, 18, p. 628.

B Brit. Med. Jour., 1905, 1, p. 1257

- Jour. Path. \& Bacteriol., 1914, 18, p. 445

T Jour. Med. Res., 1919, 34, p. 449. 
In this connection the report of MacConkey ${ }^{8}$ on some cases of food poisoning should also be recalled. This worker isolated a paratyphoid $\mathrm{B}$. bacillus from the hind limb of a rabbit, the only part of a meal which had apparently caused three cases of food poisoning and the death of a 6 months old baby. This organism was identical with the nonlactose fermenting bacteria obtained from the intestines of the baby. Certain facts presented by MacConkey make it certain that when purchased the rabbit was fit for human consumption and contamination must have taken place at the consumers' house. The possible importance of the observation of MacConkey will be considered separately more in detail.

In the light of this information it appeared profitable to investigate more carefully the spontaneous epidemic that occurred in our animal house. This study was particularly valuable in furnishing a rabbitpathogenic paratyphoid bacillus and a number of data, which assisted us materially in the analysis of the factors of immunity operative in experimental typhoid carriers of this species. We shall have occasion to refer to the organisms isolated from our epidemic in other papers and consider for the present only the symptoms, the necropsy and microscopic findings, the organism isolated, its serologic classification in the paratyphoid group and its pathogenicity for various animals.

\section{History of OUR EPIDEMiC}

Nov. 26, 1918, two lots of rabbits were received in the laboratory. One set came from a reliable dealer; his rabbits had not, during the past two years, shown signs of coccidiosis or "snuffles." The second lot was sent out by a breeder unknown to us, who had been recommended as always having been successful in raising rabbits. When these rabbits were unpacked, one female was found to have diarrhea and signs of a previous abortion. This animal was immediately isolated but had already been in intimate contact with 12 others of the same lot. This visibly diseased rabbit died Nov. 8 and was only superficially examined. No attention was paid to lung lesions and no cultures were taken. Death was attributed to a polybacterial puerperal septicemia, not uncommonly observed in rabbits closely packed and shipped in unsuitable boxes.

Dec. 8 and 9 two rabbits, that had been in contact with this animal, succumbed. Both animals had diarrhea and extensive bilateral croupous pneumonia. Cultures of paratyphoid $B$. bacilli were obtained from the lung tissues of both rabbits. The anatomic lesions did not suggest additional cultures. However, the lung lesions differed in many respects from those ordinarily encountered in our laboratory and indicated, even before we had obtained and studied the cultures, that we were not dealing with one of the common, intercurrent infections. A careful quarantine and a daily inspection of the stock animals was immediately instituted.

A fourth rabbit, rabbit 1371 , was found to be sick on Dec. 11 . It refused all food, had a purulent nasal discharge and diarrhea, and its coat was shaggy and rough. This large animal, weighing $3150 \mathrm{gm}$., was chloroformed. The anatomic lesions were typical of those commonly found in experimentally produced paratyphoid infections. Cultures were obtained on direct plating from all the organs, except the bile. The heart blood contained 720 colonies per c c.

Dec. 13 a fifth rabbit, 1372, was found dead. This animal had not been in contact with the previously mentioned rabbits and had been placed in a single

s Jour. Hyg., 1906, 6, p. 570. 
isolation cage of the infectious disease room the day before. It had anatomic lesions similar to 1371 . The intestinal sloughs were particularly well marked in the appendix region. Cultures were also obtained from all of the organs. Epidemiologically this animal was probably infected by the careless use of cleaning utensils employed in the removal of the manure. Previous to Dec. 8, when we were not as yet fully informed as to the nature of the disease, the caretaker used the same scraper on the cage holding rabbit 1372 that he had used in cleaning the section in which rabbits 2 and 3 had succumbed. It was quite obvious that only rigorous measures would interrupt the chain of continuous contact infections. Thorough disinfection of all the suspected cages with lysol and lime, sterilization of the cleaning utensils, food and drinking cups accomplished the desired result. Further cases have not occurred.

\section{Symptoms AND Lesions}

Only two spontaneously infected and several artificially infected (feeding) rabbits were available for a study of the clinical symptoms. After an incubation period of from 2 to 3 days the animals invariably had fever, the temperature being above $40 \mathrm{C}$., reaching $41.3 \mathrm{C}$. on one occasion. The hair was rough and shed more readily. In two animals a seropurulent nasal discharge was noticed. Usually they refused to eat, but drank much water. The total loss of weight varied with the individual case but was noted in every instance. There was marked pallor of the mucous membranes due to a distinct drop in the hemoglobin from 90 to $52 \%$. A few animals showed a slight hyperleukocytosis. Every animal developed severe diarrhea preceding death by several days. This symptom was frequently accompanied by a paresis of the posterior extremities and involuntary passage of urine. There was a quickened pulse, labored breathing and general prostration, a drop in temperature and convulsions signalized the approaching termination in from 4 to 8 days after the onset of the symptoms.

The gross lesions were: Emaciation was always more or less marked. The left or right anterior lobe of the lung of the spontaneously infected animals was as a rule covered with a thin layer of fibrin, the lung parenchyma was consolidated in lobar distribution or showed an infarct-like area surrounded by patchy pneumonia. The anterior portion of the left or right middle lobe showed red and gray hepatization. Also the right anterior and heart lobe were similarly affected and the pleural covering of the pericardial sac frequently showed a fine network of fibrin. The bronchi contained a small amount of grayish purulent exudate. The heart muscle was flabby and decidedly fatty. The blood was thin and sometimes of a brownish tinge. The spleen, which was dark brown, engorged, pulpy, and was enlarged, for example, to $8 \times 1.7 \times 1.5 \mathrm{~cm}$., weighing 6 to $14 \mathrm{gm}$. (The normal weight averages between $0.45-1.1 \mathrm{gm}$.) Both kidneys were enlarged, yellowish brown in color, petechiated and showed extensive parenchymatous degeneration. The mesenteric lymph nodes were enlarged, soft and juicy, and contained numerous small or large hemorrhages. Not infrequently the hepatic, peri-aortic and iliac lymph nodes were enlarged and distinctly hyperemic. The liver was increased in size and either dark or pale in color, friable and dotted with small necrotic foci of varying sizes. Along the margins large subcapsular brownish-yellow patches were noted. The gallbladder was distended by a dark, olive green, viscid bile; the wall was thin and soft; no changes were visible on the mucous membrane. In the rabbits infected by intravenous injection the gallbladder was leathery, necrotic and the bile thin or thick and purulent.

The bone marrow of the femur was a light yellowish-brown, soft or deep red and showed a few areas suggestive of necrosis. The stomach externally and internally seemed normal. Stringy, bile-tinged mucus filled the duodenum, the mucous membrane was slightly swollen and was sometimes petechiated. All 
the Peyer's patches of the jejunum were swollen, deep red, ulcerated or covered by brownish-red scablike sloughs. The contents were greenish and frothy. Sacculus rotundatus was considerably thickened; the lymphatic structure studded with numerous deep hemorrhages and small pinhead-like abscesses. The mucous membrane was covered by a glassy, slightly blood-tinged slime, and in two spontaneously infected animals consisted of extensive cauliflowerlike sloughs. In one rabbit the appendix mucous membrane was banded by broad hemorrhagic areas $0.5 \mathrm{~cm}$. in width or dotted irregularly by diphtheric hemorrhagic round or irregular sloughs. A glassy blood-tinged mucus covered the completely or partially necrotized mucous membrane. The extent of these intestinal lesions varied considerably. The artificially fed rabbits (bile method of Besredka) presented a strikingly rigid cecal or colonic wall, together with a diffuse diphtheric necrosis of the mucous membrane in contrast to the scattered ulcerations observed in the spontaneously infected animals.

Rabbits immunized with living organisms or those that had recovered from an acute infection invariably showed pinhead-like whitish or yellowish abscesses in the lymphoid tissues of the sacculus rotundatus or appendix; such findings have been recorded by Theobald $\mathrm{Smith}^{\circ}$ in rabbits infected with $\mathrm{B}$. suipestifer. These abscesses were either sterile or contained streptococci. The uterine horns of two rabbits were enlarged, contained partially dissolved putrid fetuses and an endometrium covered with yellowish necrotic areas. In one male rabbit the epididymis were edematous, hemorrhagic and showed on section numerous areas of necrosis. An orchitis was not present. Sexotropic properties apparently characterized the organism of this epidemic.

The histologic examination of some of the organs revealed changes not commonly encountered in paratyphoid infections of laboratory animals. An endarteritis and endophlebitis were noted in every tissue, not only in the spontaneously but also in some of the experimentally infected animals. Again the characteristic hyperplastic reaction regularly found in human typhoid fever was entirely absent or indicated only by a few scattered macrophages and phagocytes. These observations were unfortunately recorded only when our organisms had already lost their original virulence, and additional infection experiments were mostly unsuccessful or resulted in morbid lesions different from those seen in the spontaneous cases. A careful histologic study of paratyphoid infections should be undertaken in order to elucidate the pathogenesis of this group of organisms. Such a study is particularly desirable in the light of the recent reports of Huebschmann, ${ }^{10}$ Herxheimer, ${ }^{11}$ Dawson and Whittington, ${ }^{12}$ and others who noted not only bacteriologic but also fundamental anatomic differences between paratyphoid $B$. and true typhoid infections in man. Our incomplete data are presented in order to encourage such research on spontaneously infected animals when the occasion arises, and they should not be considered an exhaustive inquiry into this phase of the infection.

The pneumonic changes described apparently developed in three of the spontaneously infected animals as a bronchopneumonia with subsequent lobar red and gray hepatization. This associated type of pneumonia developed as a result of pronounced inflammatory processes in a large number of the interalveolar arteries and veins. The lumen of these vessels was filled with nests of fragmented and normal leukocytes of varying origin. The endothelium was loosened from the intima and the media and with the adventitia were edematous and invaded by leukocytes. In some vessels fibrin deposits and bacterial plugs

\footnotetext{
- U. S. Department of Agriculture, Bureau Animal Industry, Bulletin No. 6, 1894, p. 25.

10 Beitr. z. path. Anat. u. z. allg. Path., 1913, 56, p. 514.

11 Berl. klin. Wchnschr., 1916, 53, p. 648

2 Quart. Jour. Med., 1916, 9, p. 98.
} 


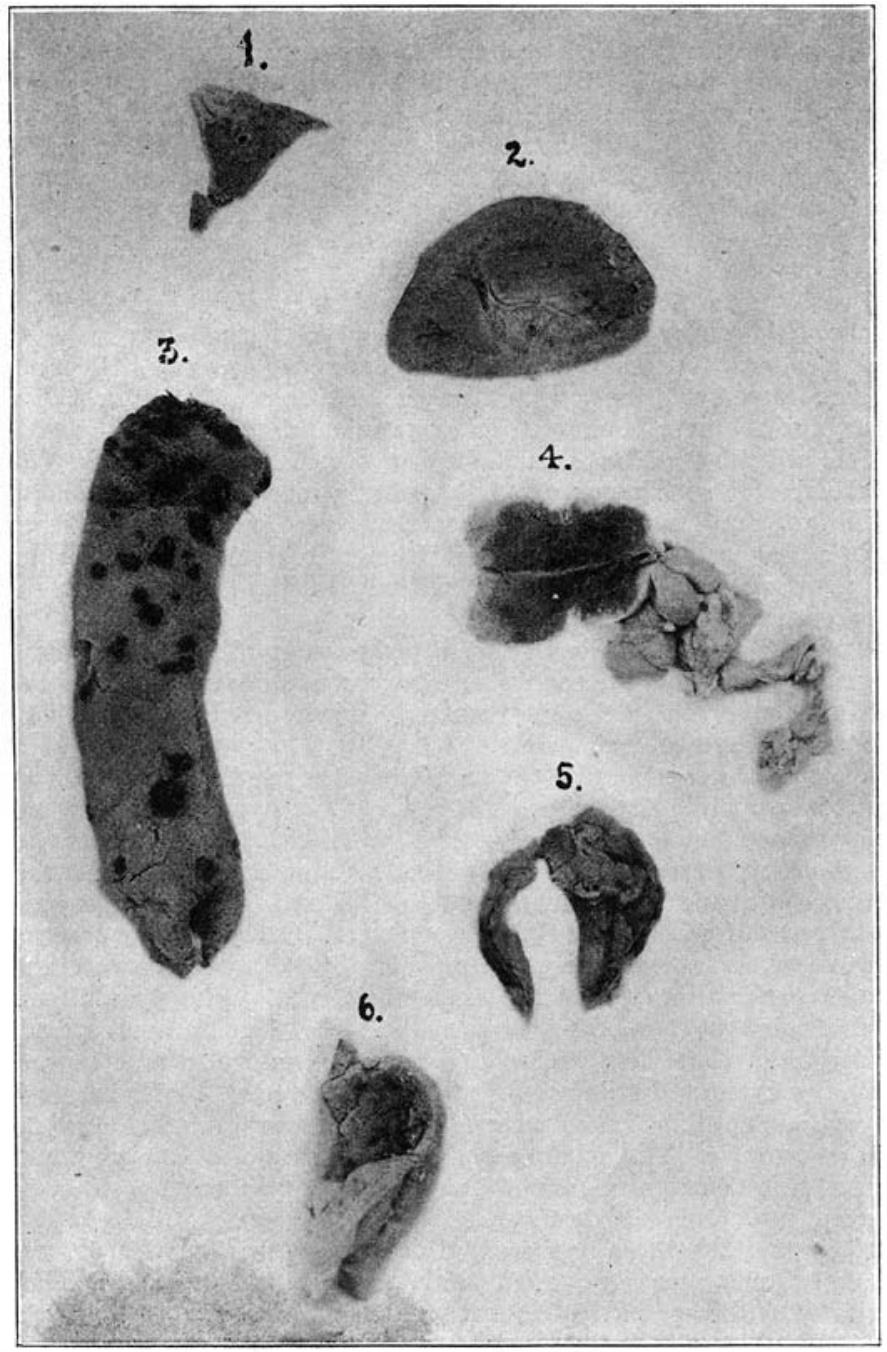

Fig. 1.-Anatomic lesions in rabbit 1371: (1) transverse section of left anterior lobe; (2) kidney; (3) appendix vermiformis opened; (4) mesenteric lymph nodes; (5) incised Peyer's patch; (6) sacculus rotundatus opened, hemorrhagic slough on mucous membrane. 
were recognized. Surrounding these inflamed vessels were alveoli containing coagulated albuminous material, fibrin, red cells or leukocytes or even infarctedlike areas. Certain complexes of lobules showed incipient or advanced stages of necrosis, and these areas fused with the bronchopneumonic patches of the lung tissue. The extensive involvement of the terminal bronchi, which were filled with leukocytic plugs extending into the neighboring air cells, suggests that these animals suffered in all probability previous to the paratyphoid infection from the common bronchitis due to $\mathrm{B}$. cuniculisepticus. It should, however, be remembered in this connection that Dawson and Whittington, Herxheimer and particularly Klein and Torrey ${ }^{13}$ consider bronchitis and bronchopneumonia as important anatomic lesions in human paratyphoid B. infections. Artificially infected rabbits, however, failed to show similar pulmonary changes, which supports the contention of a mixed infection.

The liver sinusoids of all the animals spontaneously or artificially diseased were moderately engorged with blood. The peripheral liver cells contained large fat droplets. Numerous areas consisting of necrosed liver cells and accumulated leukocytes in various stages of fragmentation or typical toxic "pseudo tubercles" were common. In some instances these focal necroses were around the central vein, in others they involved several lobules. Invariably the portal veins or spaces contained thrombotic material and exhibited definite zones of infarction. We have the impression that the necrosis is the end result of a toxin action and not the outcome of embolic blocking of the sinusoids by leukocytes or even splenic cells. Our observations are in this connection quite in accord with those reported by Gruber ${ }^{14}$ and Wagner and Emmerich. ${ }^{15}$ Bacterial clumps were occasionally seen in the areas of focal necroses. Typical "lymphomas," consisting of macrophages, were not observed. The gallbladder wall was only involved in the intravenously injected animals. The importance of this fact will be discussed below.

The follicles or germ centers of the spleen were usually enlarged and showed a distinct toxic hyaline degeneration ("eosinophilic hyperplasia") or were completely submerged in the pulp changes. The pulp was engorged with red cells or pigment débris partially inclosed in cells. Numerous necroses, thrombi or fibrin, phagocytic and desquamated pulp cells and nuclear fragments in the splenic sinuses formed a complicated picture. Macrophages were not found, but nests of leukocytes well preserved or partially disintegrated were not uncommon. The cortical sinuses of the mesenteric and portal lymph nodes were slightly distended by leukocytes and hyaline-like débris; the central sinuses, particularly near the hilum, were packed with red cells and large red cell carrying phagocytes and polymorphonuclear leukocytes. No hyperplasia of the follicles or the medullary cords were noted in the large number of nodes examined for this purpose. The spontaneously infected rabbits showed, however, distinct endarteritic changes in several small arteries and patches of more or less marked necrosis in the follicles.

In the jejunum dilatation of the blood vessels and catarrhal hypersecretion of the mucous glands were the most striking changes recorded. The Peyer's patches showed either extensive hemorrhages or complete necrosis of the mucous membrane covering the lymphatic tissue. These changes were accompanied by leukocytic infiltration, at times forming a distinct zone of demarcation. Invariably the submucosal blood or lymph vessels were plugged with leukocytes or thrombotic material. The necrotizinz diphtheria inflammatory processes of the sacculus rotundatus, appendix and ileum were confined to the

\footnotetext{
${ }^{13}$ Am. Jour. Med. Sci., 1920, 159, p. 546.

14 Centralbl. f. Bakteriol., O., I, 1916, 79, p. 1.

15 Ibid., 1916, 77, p. 301.
} 
intestinal wall provided with lymphatic tissue. In these places a confluent mass of necrosis, together with an extensive hemorrhage and little or no fibrin, covered an area of cellular degeneration and disintegration extending to the oedematous muscularis.

The diphtheric changes in the colon of the rabbits infected by first feeding bile and following with the ingestion of large amounts of paratyphoid bacilli resemble those of epidemic dysentery. A phlegmonous infiltration of the entire wall of the mucous membrane was covered by a layer of fibrin and necrotic tis sue.

The mucous membrane of the appendix in one rabbit was in its entire distribution necrotic and appeared as a faintly stained mass resting on a circular zone of demarcation just above the lymphatic tissue layer. In some of the immune or recovered animals the follicles of this portion of the appendix contained large areas of necrosis and leukocytic aggregations forming a micro abscess. There were changes in the regional capillaries, but not as marked as those described in the lung and the mesenteric lymph nodes. The lesions are unquestionably different from those reported in human typhoid. They develop in the lymphatic tissues of the intestinal canal but not on the basis of a hyperplastic reaction. Hemorrhages and necroses, apparently the result of vascular injury, predominate and in this respect the anatomic process of "rabbit typhoid" finds an analogy in human paratyphoid or in hog cholera. As far as the appendix is concerned, the lesions remind the observer strongly of those seen in acute human appendicitis; certain stages are strikingly similar to those described by McMeans $^{16}$ in his able article on experimental appendicitis.

Some sections of the bone marrow exhibited hemorrhages, leukocytic accumulations and in one instance small scattered areas of necrosis. The myocardium of one rabbit (1415) showed extensive fatty degeneration of the muscle fibers; in the majority of animals indications of a beginning interstitial myocarditis were visible. The blood vessels of the epicardium and myocardium were dilated and frequently possessed nests of leukocytes.

Hyaline thrombi in the glomerular capillaries, hemorrhages, irregular degeneration of the tubular lining, perivascular edema and albuminous tufts were noted in the kidneys. The suprarenals were normal. In one male rabbit the spermatic vein contained thrombotic material and leukocytic débris with adjacent necrosis of a portion of the epididymis. The uterus of two animals contained necrotic embryonic tissue and the endometrium possessed all the signs of a marked inflammation with necrosis. Clinically, as well as anatomically, this spontaneous paratyphoid infection in rabbits differed in no respect from the disease constantly observed and carefully described for this species inoculated with various representatives of the paratyphoid-enteritidis group, by Smith and Moore, ${ }^{17}$ Karlinski, ${ }^{18}$ Raccuglia, ${ }^{19}$ Ordway, Kellert and Huested, ${ }^{20}$ Okubo," and others. The descriptions given by these writers cover our observations in every detail as far as the gross morbid lesions are concerned, but we are unable to find the endothelial hyperplasia and the typhoid-like microscopic changes mentioned repeatedly by Ordway and his associates. Vascular changes and necroses predominated in the tissues of our animals. It is not unlikely that the high virulence of the organism and the comparatively short duration

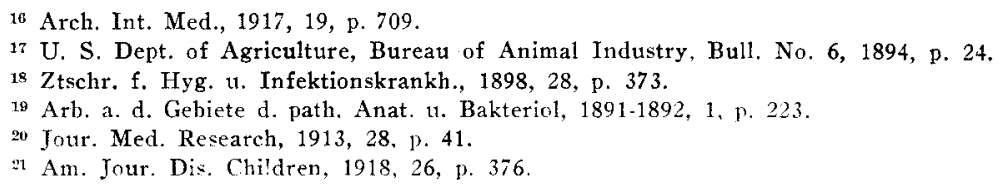


of the disease in our rabbits in comparison to the prolonged course of the infection in the observations of Ordway are in part responsible for the differences. However, in the light of recent knowledge on the morbid anatomy of paratyphoid fever in man, the microscopic findings have more than academic interest and urgently deserve additional investigations on suitable material. Such a study appears to us particularly profitable, when we appreciate that the information concerning the factors of pathogenesis, toxigenesis and immunity of the typhoid-paratyphoid group must indeed be considered meager.

\section{The ORganism}

Impressions of tissue sections or fragments and direct quantitative plating of the secretions of the spontaneously infected rabbits on brilliant green-eosineagar or peptic digest endo-agar gave a pure growth of abundant colonies of a gram-negative, motile bacillus. The heart blood of one rabbit (1371) produced 720 colonies per c c. From the intestinal content, the wall of the jejunum, ileum, sacculus rotundatus and cecum, the liver, the spleen, the lung, etc., a practically pure and profuse growth of the same organism was obtained. The bile did not contain viable bacteria on direct plating of $1 \mathrm{cc}$; on enrichment in broth paratyphoid-like organisms could be demonstrated.

The isolated organisms fermented the following carbohydrates, with the formation of gas, glucose, maltose, levulose, mannite, galactose, dulcite, mannose, arabinose, sorbite, rhamnose, and xylose, but had no effect on lactose, saccharose, dextrin, inulin, salicin, raffinose, dextrin and adonite. The fermentation of inosite was slow; acidification was noted only after 48-60 hours' incubation. In lead acetate agar, there was a browning of the medium at the end of 24 hours, and glucose serum water, according to Krumwiede, was reduced. Neutral red glucose agar was split by gas production and reduced. In bromcresol-purple-milk, the reaction at the end of 24 hours was slightly acid, and remained so for 48-72 hours. After the fourth day the medium turned gradually alkaline and at the end of 20 days was slightly saponified. Gelatin was not liquefied in 30 days and no indol was produced in Difco or Witte's peptone solution. The organisms are highly resistant to brilliant green. The final acidity in glucose diabasic phosphate-peptone solution was $\mathrm{P}_{\mathrm{H}} 4.8-5.0$ after 48 hours and $P_{H} 5.0$ in mannite after the same period of incubation. The biochemical reactions just mentioned place the organisms isolated with the $B$. schottmülleri type of the colon-typhoid group. The behavior in carbohydrate mediums followed the type III reaction described by Winslow, Kligler and Rothberg in 1917.

\section{Aggletination}

The strains isolated from the various tissues were readily agglutinated in the first generation by a polyvalent human paratyphoid B. (B. schottmülleri) serum in a dilution of 1:500 and were not influenced by a highly potent $B$. enteritidis serum in dilutions above $1: 10$. Subsequent serologic tests were therefore conducted with the representatives of the paratyphoid-suipestifer group. The technic employed in order to classify the rabbit organism was identical to the one recently described by one of us. ${ }^{22}$

Specific antiserums against paratyphoid B. strains of rodent, avian, porcine and human origin were obtained by subcutaneous injections of living organisms in progressive amounts varying from $0.01 \mathrm{cc}$ to $0.2 \mathrm{cc}$ of a 24-hour old broth culture at weekly intervals. This mode of immunization produces characteristic local necroses and abscesses.

\footnotetext{
22 Feusier and Meyer: Jour. Infect. Dis., 1920, 27, p. 185.
} 
According to the chart the bacillus (lung strain 2 and the heart blood strain 1371 behave in an identical manner) is by direct agglutination a paratyphoid B. closely related to the rodent type of this large group. It has also been definitely proved that the strain does not belong to either the human, avian (true paratyphoid strains not B. sanguinarum or B. pullorum) or porcine paratyphosus "B" group. A rabbit antiserum prepared with our organism possessed group agglutinins for the guinea-pig-paratyphoids, for 2 avian and for 2 swine typhus strains. A rabbit and a guinea-pig serum produced with a guinea-pig-paratyphoid strain exhibited group affiliations to our bacillus and to a number of guinea-pig organisms isolated from different epidemics. The striking group agglutination reactions among the avian paratyphoids by these and other serums are noteworthy. A culture isolated by $\mathrm{Dr}$. N. S. Ferry from a rabbit, and sent to us as a representative of the paratyphoids found by him in these animals, behaved more like a member of the avian than of the rodent group. The mutual cross agglutination among the guinea-pig and the avian paratyphoids is strongly suggestive of a close relationship. We are not in possession of the strains employed by Krumwiede, Valentine, and Kohn, and we can therefore not state conclusively that our organism belongs to their group.

Absorption tests failed to classify our organism further in the animal paratyphoid group. Antigenically the rabbit paratyphoid strains are members of the animal paratyphoid group, which can be readily distinguished by absorption tests from the true human B. paratyphosus, "B" or B. schottmülleri the causative organism of human paratyphoid fever. Further subgrouping of the animal paratyphoid into rodent, avian or porcine groups may be accomplished by direct agglutination, but a more conclusive classification is impossible by the customary absorption technic.

The hog cholera bacillus or B. cholerae suis represented in our collection by a number of strains designated as $B$. suipestifer and $B$. voldagsen and B. typhi suis do not, according to the tests of others and our own, belong to the rabbit group of animal paratyphoids, but are to be classed in a group by themselves. Some paratyphoid strains, of porcine origin, the so-called swine typhus culture I and II are related to our rabbit organisms. These observations support the recently formulated conclusion of Ten Broeck ${ }^{23}$ relative to the existence of a distinct variety of paratyphoids common to animals and justify the acceptance of the terminology proposed by him. The organism could be designated as a member of the "B. aertryckei-group." Unquestionably further study in this large group of luacteria will, in our opinion, reveal additional subgroups with distinct host relationship.

\section{Pathogexicity}

The rapid progress of the epidemic obviously suggested a virulent strain of paratyphoid bacilli. As transmission from rabbit to rabbit in all probability resulted by way of the intestinal tract in form of a feeding infection, it was of interest to determine the approximate number of bacteria necessary to cause a fatal issue in these animals. It is generally stated and quite recently emphasized by Besredka ${ }^{24}$ that the rabbit is nonsusceptible ("tout à fait invulnérable") against massive doses of paratyphoid or typhoid bacilli introduced into the digestive tract. This statement deserves some modification. Occasionally recently isolated, parasitic animal and human strains were encountered which can produce typical lesions and generalized infections when administered by

$\because 3$ Jour. Exper. Med.. 1920, 32, p. 19.

"Bull. de l'Inst. Pasteur, 1920, 18 p. 123. 


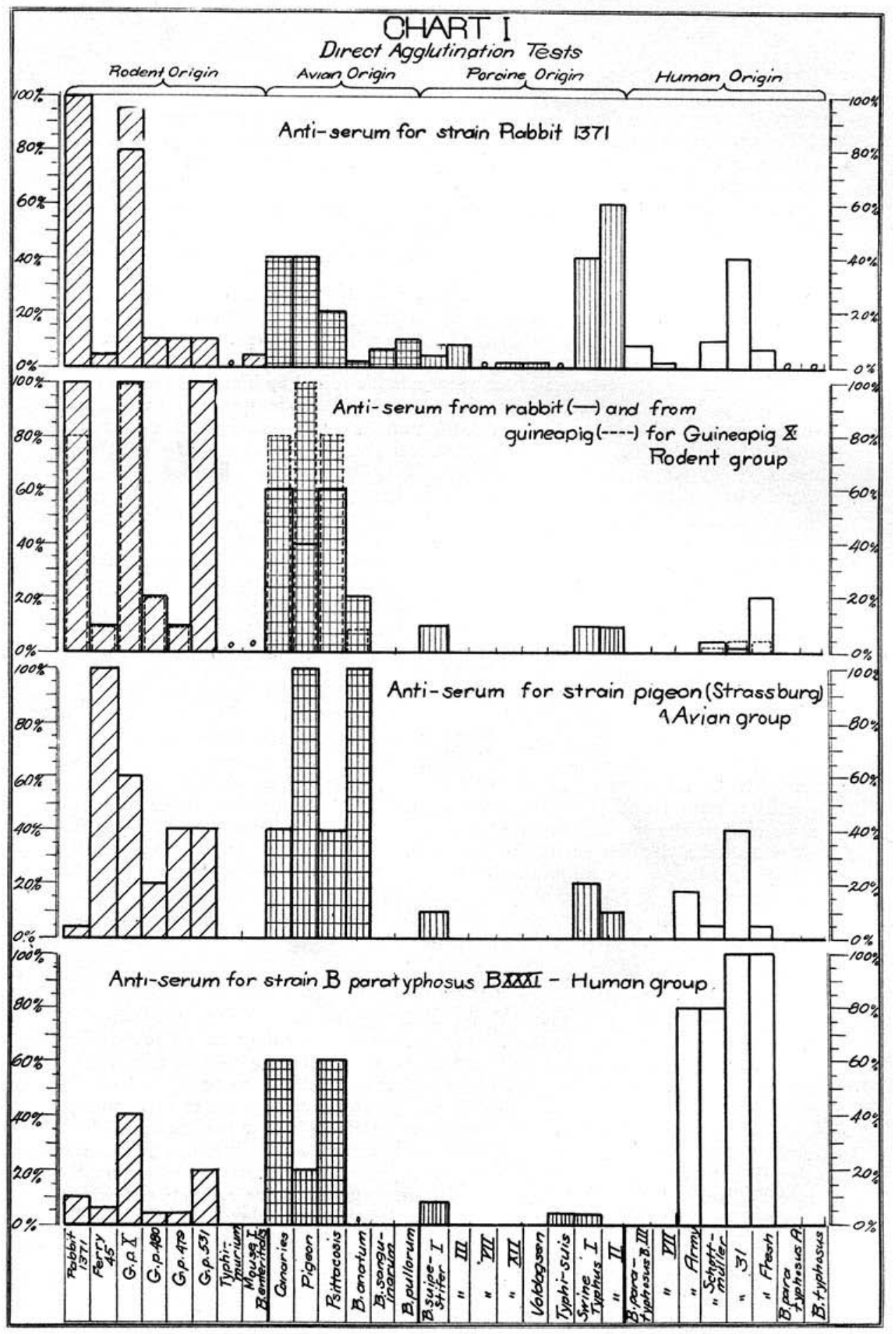


mouth. From a comparative standpoint it was also important to test our parasitic and saprophytized organism on other laboratory animals, particularly mice and guinea-pigs.

Inoculation Experiments.-Mice and guinea-pigs inoculated subcutaneously with $0.1-0.01 \mathrm{c} \mathrm{c}$ of a 24-hour broth culture succumbed in from 12 to 24 hours. The inoculated organism was found in the heart blood and in the organs. Even 15 months after isolation the strain retained approximately the same degree of virulence.

Subcutaneous inoculations of $0.1-0.01 \mathrm{cc}$ of a 24 -hour broth culture $2 \frac{1}{2}$ months after isolation produced in a large number of rabbits more or less extensive necroses and local abscesses. A rise in temperature for several days, loss of appetite, perhaps temporary diarrhea and an enlargement of the regional lymph nodes accompanied the local processes. Ten days after the inoculation the blood serum of such rabbits agglutinated the paratyphoid bacillus in dilutions from 1:400-1:800. Two additional injections produced local lesions only and usually immunized the animals completely as will be shown in another paper. Judging from the observations of Ten Broeck ${ }^{25}$ with B. suipestifer, our bacillus possesses in comparison a low virulence on subcutaneous application and our findings lend little support to the supposition that fleas or other insects could be considered as factors in the epidemic.

The intravenous inoculation, on the other hand, was fatal in comparatively snall doses in from 2-10 days. The number of recently isolated cultures necessary to kill adult rabbits of different litters and age varied between 200,000 and 700,000 organisms in broth cultures of different compositions and reactions. The six month old strain was pathogenic in doses of $1-2$ billion and after one year this number of bacteria was fatal only in young rabbits. Irrespective of the variation in virulence the clinical symptoms, following a distinct incubation time and the anatomic lesions of a septicemia, were practically the same. Of particular interest are the findings of a diphtheric or even necrotizing cholecystitis with positive cultural results in every nonimmunized rabbit which succumbed to the intravenous injection.

These experiments only demonstrate that the rodent paratyphoid bacillus possessed for laboratory animals an average virulence, which differed in no respect from the one ordinarily noted with recently isolated human or animal paratyphoid strains.

Feeding Experiments.--In the feeding experiments on coccidiosis-free rabbits the cultures were grown on agar slants and in peptic digest broth. Only cultures 24 hours old were used. The data obtained indicates that the ingestion of 2 billion recently isolated organisms produced the disease with characteristic lesions. When the strain was 6 months old the results became irregular with amounts as high as 8 billion, and when 12 months old even 1,600 billion were innocuous in a large series of animals. At the time of writing enormous doses $(5,000$ billion in milk) are necessary to produce an infection by feeding. Even $20-50$ billion organisms are required to confirm the observations of Besredka ${ }^{20}$ of feeding bile preceding the administration of organisms of the dysenteryparatyphoid group. The virulence of the organism has progressively degenerated and infected rabbits shedding these paratyphoid organisms can with impunity be mixed with susceptible ones. On the other hand, the epidemiologic data presented indicated a high original virulence which unquestionably

25 Jour. Exper. Med, 1918, 28, p. 759.

26 Ann. de 1'Inst. Pasteur, 1919, 33, p. 557. 
increased by passage from animal to animal, probably in a manner somewhat similar to the one described by Ten Broeck. ${ }^{27}$

Even guinea-pigs were endangered, though spontaneous cases were not observed by us. Ingestion of 250,000 organisms was fatal for guinea-pigs varying in size from $400-500 \mathrm{gm}$. in from 4-8 days, using the recently isolated culture. The necropsy findings differed in no respect from those commonly noted in rodent paratyphoid or pseudotuberculosis. The virulence of cultures 6 to 12 months old diminished similarly for guinea-pigs as for rabbits, and larger doses were necessary to produce an infection. Daily feeding of a whole growth of an agar slant (culture 18 months old) on minced carrots for 3 weeks produced a chronic disease with positive cultural findings in the spleen, mesenteric lymph nodes and liver. It was noted that white mice that accidently had access to the remains of the carrot mixture succumbed to an acute infection (10-12 days), with typical lesions and positive cultural findings in all organs. At least for this species of animal the organism had retained a considerable degree of virulence.

The feeding experiments on rabbits furnished a series of observations that appear to be of considerable importance and therefore deserve some consideration. Contrary to our findings in the intravenously inoculated rabbits in which a diphtheric or necrotized cholecystitis with positive paratyphoid bacilli findings were recorded, the rabbits fed and successfully infected with the same organism of the same age did not show a cholecystitis. Invariably the cultures obtained from the bile were sterile. These results are fully in accord with those described for the spontaneous cases. We also recall in this connection that the recent statistics of Hübener ${ }^{28}$ and Herxheimer ${ }^{11}$ on acute paratyphoid fever infections in man fail to mention pathologic changes in the gallbladder. One is probably justified in assuming that in their cases, at least, this organ was not diseased. These facts may contribute some information concerning the pathogenesis of cholecystitis due to organisms of the typhoidparatyphoid group in rabbits free from coccidiosis or other infections of the liver. One fact stands out preeminently, namely, irrespective of the number of paratyphoid bacilli in the blood and tissues at the time of death, the animals that received their infection by way of the intestinal tract did not show a cholecystitis nor even bacilli in the bile so perfectly suited for their proliferation. The gallbladder wall may on enrichment in broth be found to be infected, which is not surprising when the blood stream is teeming with specific organisms.

It will be the purpose of another series of papers to discuss in detail the manner in which typhoid bacilli, for example, reach the gallbladder and bile in rabbits, but we can already state that an ascending invasion via the common and cystic duct has not been observed in our studies. On the other hand, there is conclusive evidence at our disposal which indicates that the infection is descending. Typhoid or paratyphoid bacilli inoculated intravenously in certain doses, which vary according to the virulence of the organisms, the size of the animal and other factors to be considered elsewhere, appear in the common duct bile in the first 10-15 minutes after the injection. The bacteria reach the bile by way of the biliary passages and proliferation in this secretion or the constant feeding of the same from liver foci will lead to a more or less prolonged sojourn of the organisms with secondary inflammatory involvement of the gallbladder wall. In our experience another route of bile infection is occa-

${ }_{27}$ Jour. Exper. Med., 1917, 26, p. 437.

28 Fle:schvergiftungen und Paratyphusinfektionen, 1910, p. 118. 
sionally noted in rabbits, namely, the embolic invasion of the capillaries of the gallbladder wall. This mechanism of invasion is particularly common when virulent strains in large doses are used. The histologic picture of the gallbladder wall of the intravenously inoculated rabbits in our pathogenicity series suggested such a hematogenous infection as the most likely mode leading to the diphtheric or necrotized cholecystitis. Bacterial thrombi are found in the capillaries at the base of the fundus of the gallbladder mucous membrane. These foci are surrounded by areas of necrosis or leukocytic infiltration. Such portions of the mucosa may be covered by an intact epithelium. In the majority of cases, however, the complete destruction of the entire mucous membrane, muscularis and serosa does not permit an insight into the cycle of events leading to the complete destruction of the gallbladder. The difference in the gallbladder lesions between the spontaneously infected, the experimentally fed and the intravenously inoculated rabbits may therefore be readily explained as follows: Paratyphoid bacilli intravenously introduced rapidly accumulate in large numbers in the liver capillaries; the gallbladder as an appendix of this organ and connected by accessory portal veins (Violle ${ }^{20}$ ), receives a proportional share in its small blood vessels. In the parenchyma of the liver the characteristic necroses and cellular proliferation develop as a result of the multiplication of the retained bacteria; in the gallbladder wall a similar process leads to a diphtheric necrosis of the mucous membrane with subsequent invasion of the bile. The latter may also receive bacteria simultaneously from the biliary passages. Intensive proliferation in this secretion leads to additional destruction of the mucous membrane from within and in turn may cause complete necrosis, even beginning perforation of the gallbladder wall. In the spontaneously infected or in the fed animals the number of organisms that reach the liver is comparatively small and the invasion gradual. The protective properties of the liver (also seen in immunized rabbits inoculated intravenously) prevents the indirect passage of paratyphoid bacilli from the hepatic veins to the biliary capillaries. A few bacteria reaching the bile are probably rapidly discharged in the hepatic duct bile, which is continuously flowing as result of the toxic enteritis, and in all probability no organisms reach the cystic bile where multiplication is most suitable. This reconstruction of the events is borne out by the cultural studies in this and other series of animals studied for the same purpose. The gallbladder bile, the duodenal and jejunal contents were free from paratyphoid bacilli. Such bacilli were found only around or below the diphtheric or ulcerated agminated follicles and in the region of the sacculus rotundatus and appendix. On the other hand. the gallbladder wall of the fed or spontaneously infected animals was found to contain a few paratyphoid bacilli; this is not surprising when we realize that on one occasion 720 organisms were present in 1 c.c. of heart blood. A direct, heavy invasion of the gallbladder wall and its blood vessels was made impossible on account of the gradual infection of the liver from the portal system. Consequently, the formation of bacterial emboli primarily responsible for the diphtheric inflammation was eliminated. These observations furthermore show that in the rabbit at least a portal septicemia with rabbit pathogenic virulent and invasive paratyphoid bacilli does not necessarily lead to a cholecystitis or to an infection of the bile. On the other hand, the intravenous injection of the same organism in comparatively small numbers catses a hematogenous invasion of the gallbladder and bile by way of the biliary passages on one hand and by way of the capillary thrombi in

29 Ann. de l'Instit. Pasteur, 1912, 26. 1. 384. 
the wall on the other. In case the animal has lesions of coccidiosis or the organism possesses specific selective organotropic properties for the gallbladder, as was shown by Fraenkel and $\mathrm{Much}^{30}$ localization in this organ may occur following any method of infection. These facts have in our opinion a bearing on the typhoid-paratyphoid carrier problem in laboratory animals, which we will discuss elsewhere more in detail.

\section{Toxin Production}

Smith and Ten Broeck ${ }^{30}$ observed that bacteria of the typhoid-paratyphoid group are capable of producing highly potent toxins for rabbits in peptonized sugar-free veal broth plus $0.1 \%$ glucose incubated in shallow layers. One of us (K. F. M.) several years ago conducted a large number of experiments on dogs and rabbits with the toxic filtrates from B. abortus equinus prepared according to the procedure given by these writers. The results were rather irregular and at that time it was impossible to investigate the factors responsible for the inconstant results. A series of tests conducted with the paratyphoid bacilli isolated from the rabbit apparently offer an explanation for the failures recorded. It was found that aside from the depth of the layer of fluid in which the cultures were grown, namely, not more than $2 \mathrm{~cm}$., the reaction of the medium is an important factor. We originally prepared the substratums for the production of toxic filtrates strictly according to the formula of Smith and Ten Broeck with Witte's peptone, adjusting the fluid to an initial reaction of $1 \%$ acid to phenolphthalein. The final product had a $\mathrm{H}$-ion concentration of $\mathrm{P}_{\mathbf{H}}$ 7.2-7.4. Several batches of mediums with this reaction were tried. Invariably the toxic filtrates of 7-day old cultures, inoculated in doses of $1 \mathrm{c} \mathrm{c}$ per kilogram of weight, produced temporary restlessness, labored breathing followed by slight stupor, passage of urine and feces and loss of weight of from 75-100 gm., but never death. Experiments with other toxins and particularly the generally known fact that diphtheria toxin is only produced at a $\mathrm{P}_{\mathrm{H}}$ of from 7.8-8.4 suggested some experiments with mediums adjusted to such a reaction. Powerful toxic filtrates, even from 48 -hour cultures, were obtained in a broth with a $\mathrm{P}_{\mathbf{H}}$ of 8.4. For illustration of this statement, 'we cite in detail one experiment:

Rabbit 1749 , weighing $2,350 \mathrm{gm}$., received at $2: 30 \mathrm{p} . \mathrm{m} ., 2.5 \mathrm{cc}$ of filtrate of a 72-hour old broth culture of paratyphoid bacilli 1371 (initial reaction of medium $\mathrm{P}_{\mathbf{H}}$ 8.4) into an ear vein. At 4:30 was drowsy and breathing labored with a snuffling noise, hind leg extended, eyes dull and partially closed. At 9 p. $\mathrm{m}$. the rabbit was found dead. The necropsy showed hemorrhages in the abdominal and pelvic lymph nodes, engorgement of the liver, and spleen, petechiae on the serosa and mucous membrane of the stomach and the duodenum. There were $2 \mathrm{cc}$ of fluid in each pleural sac; the lung was slightly congested and moist; there were intense congestion in the trachea and petechiae on the pericardium; the blood was thick and tarry.

The course of the intoxication and the necropsy findings were practically identical in all the animals injected with these toxins and in a general way differed in no respect from the description given by Smith and Ten Broeck and Mulsow ${ }^{31}$ for various representatives of the typhoid-paratyphoid group.

In connection with some other problems, we were interested in the possibility of producing toxic organ filtrates according to the procedure of $J$. T.

\footnotetext{
so Jour. Med. Research, 1914, 31, p. 523.

3. Jour. Infect. Dis., 1919, 25, p. 135.
} 
Parker. ${ }^{22}$ This writer demonstrated that the liver of rabbits inoculated with cultures of B. typhosus or B. prodigiosus under certain conditions contain a toxic substance extractable with salt solution. When the toxic extracts are injected intravenously into normal rabbits these animals develop symptoms resembling those of anaphylactic shock and succumb. We have confirmed these observations with the $B$. typhosus, but thus far we have been unable to obtain toxic liver extracts of rabbits injected with one half to one slant of our paratyphoid bacillus. Even by producing the most favorable conditions by rendering the animal very sick in 6-10 hours after the injection of one half slant of a young culture no liver poison was elaborated that would kill small rabbits in the chosen dose of $10 \mathrm{cc}$ of extract. Our paratyphoid bacillus does, however, behave in a similar manner to a number of other paratyphoid strains tested by the same technic. Certain immunity tests to be reported elsewhere suggest that the toxemic manifestations of paratyphoid infections in rabbits are different from those constantly observed in these animals injected with typhoid bacilli. A progressive septicemia with a high bacterial count of the blood and tissues precedes the death of paratyphoid rabbits. On the other hand, the fatal issue in rabbits injected with typhoid bacilli is frequently accompanied by a comparatively low bacterial blood and tissue count. It is not unlikely that the greater bactericidal power of the blood and tissues of the rabbit for typhoid bacilli is responsible for the rapid destruction of viable bacteria and the production of a large amount of poison which causes the death of the animals. These bacteriolytic forces are apparently not operative to the same degree in paratyphoid infection in rabbits, and poisons are not elaborated in the tissues; or the liver is able to neutralize large amounts of toxin as they are formed, and a quantity which exceeds the detoxicating threshold is never produced. In both instances, the poison naturally cannot be demonstrated in the salt extracts. Whether these interpretations are the correct ones or whether they only represent one phase of a more complex mechanism is at present the subject of a more extensive investigation.

\section{GENERAL SUMMARY}

The foregoing study demonstrates the occurrence of spontaneous paratyphoid $B$. infections in rabbits. Unfortunately it was impossible to determine the origin of the disease; the dealer supplying the infected rabbit, which initiated the epidemic described, had disposed of his stock when the nature of the malady was properly established. It would have been of considerable practical importance to have known whether the existence of carriers was responsithle for the development of the acute cases. The possibility that certain types of abortion in rabbits may be caused by paratyphoid bacilli and that animals in such a condition may disseminate the infection cannot be dismissed in the light of our observations. It must also be the question to be decided whether or not the causative strain was of guinea-pig or of mouse origin. The fact that the dealer did not raise guinea-pigs or mice and the extreme rarity of paratyphoid $B$. infections in rabbits in 
contrast to the rather ubiquitous distribution of this malady in guineapigs and mice, does not lend much support to this conception. Rabbits are frequently exposed to psetudotuberculosis of guinea-pigs. Repeatedly diseased animals have been introduced into our cages, and before the infection was recognized, numerous rabbits have been exposed to their discharges without causing a paratyphoid epidemic. Moreover, the agglutination tests conducted with the strains isolated from a series of different guinea-pig epidemics in our laboratory failed to indicate a close relationship to the rabbit-paratyphoid organism. The determinations of the source of paratyphoid infections must therefore be reserved for future investigations.

Our observation, which shows how readily an epidemic among laboratory rabbits may get started, should be a lesson to all laboratory workers. It is obvious that careful necropsy and complete cultural examination should be performed on all stock animals, even if the circumstances of their death suggest only an accidental infection. It has been our policy to keep complete records of the origin of the animals received and to place the same in experiments only after a strict quarantine in isolated cages for at least two weeks.

This discussion cannot be closed without a brief consideration of the relationship of our paratyphoid organism to so-called food poisoning. Although MacConkey ${ }^{8}$ reports cases of food poisoning in which rabbit meat was involved, it is definitely shown by the serologic tests that our bacillus does not antigenically belong in the human paratyphoid or B. schottmülleri group. Yet the ability of this organism to evolve a potent toxin should be considered in any attempt to explain outbreaks of food poisoning in which rabbit flesh is a part of the suspected meal. Even if the reports on meat poisoning caused by rabbit meat are limited to the one by MacConkey, it would be most unwise to say that this food cannot cause disease in man and to use this as an argument for the safety of man against the animal paratyphoid bacilli. Individual susceptibility may play an important rôle and a nonpathogenic organism may be readily transformed into a potent factor of gastro-enteritis. 